\title{
Trauer um Lutz Damerow
}

\section{Blanke}

Online publiziert: 4. Juni 2021

๑ Der/die Autor(en) 2021

Wir trauern um Dr. Lutz Damerow. Er verstarb am 19. April 2021 nach langer und mit großer Geduld ertragener Krankheit - vor seinem 60. Geburtstag. Herr Damerow stammte aus dem Obstbaugebiet Werder/Havelland im Osten Berlins in der ehemaligen DDR. Vor seinem Diplomstudium an der Ingenieurhochschule Berlin-Wartenberg absolvierte er eine obstbauliche Lehre. Nach dem Diplom im August 1988 promovierte Lutz Damerow im Rahmen eines Forschungsstudiums zunächst an der Ingenieurhochschule Berlin-Wartenberg. Im September 1991 schloss er seine Promotion zum Thema „Das Remissionsverhalten (Reflexion) von Apfelfrüchten“" an der Landwirtschaftlich-Gärtnerischen Fakultät der Humboldt-Universität zu Berlin ab.

Nach dem Fall der Mauer realisierte Lutz Damerow seinen Traum der neuen Freiheit und fuhr mit dem Auto entlang der berühmten Küstenstraße in Kalifornien zu seinem Cousin. Prof. Dr. Kromer holte ihn 1991 an die Universität Bonn in das Institut für Landtechnik. Neben den Vorlesungen übernahm er die Verantwortung für die aufwendige Werkstatt mit vielen Aufträgen und Projekten. Dort konstruierte er mit seinem Kollegen die mechanische Ausdünnmaschine, für die die beiden Projektleiter 2006 in Berlin mit dem Haymarket Innovationspreis im Gartenbau (heute Taspo-Stiftung) ausgezeichnet wurden.

Lutz Damerow entwickelte als Erster eine Hagelnetz-Abwickelmaschine, deren Bauplan im Internet frei zugänglich ist. In weiteren Projekten leistete er Pionierarbeit wie bei der Baumerkennung, Ernteschätzung und Frostabwehr bei Apfel sowie Technik für Feinsämereien, Unkrautbekämpfung

\footnotetext{
M. Blanke ${ }^{1}$

mmblanke@uni-bonn.de

1 Rheinische Friedrich-Wilhelms-Universität Bonn Bonn, Deutschland
}

mit heißen Pflanzenölen und Erkennung von Blattläusen auf Gelbtafeln - aufgrund des Schwierigkeitsgrades oft an den Grenzen der Industriepartner. In vielen Bereichen war Herr Damerow ein Vordenker und Mastermind. Im HortInnova Beratungsteam des Ministeriums leistete er hervorragende Arbeit. Er war unter den Kollegen der Community, insbesondere unter den Gartenbautechnikern ein sehr geschätzter und liebenswerter Kollege und guter Freund. Er war bescheiden, so dass trotz seiner Mitwirkung nicht immer sein Name auftauchte. Sein Leben war von der Arbeit geprägt, die er mit Hingabe zu seinem Hobby machte.

Lutz Damerow war weltoffen für alle Kulturkreise, insbesondere USA und Asien bzw. China und Thailand, mit regelmäßigen Forschungsaufenthalten an der CAU Peking und er betreute engagiert Stipendiaten aus diesen Ländern in Bonn. Auf europäischer Ebene nahm er regelmäßig an den Treffen der EUFRIN Arbeitsgruppe Blütenausdünnung und in Übersee an den Precision Agriculture Treffen in den USA teil und war Mit-Organisator des EUFRIN Treffens in Bonn/ Ahrweiler am DLR in 2017.

Dr. Damerow verfügt über zwei Patente und veröffentlichte über 60 Publikationen in internationalen, referierten und teilweise hochkarätigen Zeitschriften wie Plant Science, Biosystems Engineering und Precision Agriculture mit Beiträgen zur Alternanz, Reflexionsfolien und Ernteschätzung beim Apfel.

Sowohl in der Deutschen Gartenbaulichen Gesellschaft (DGG) als auch im

KoGa Lenkungsausschuss Dr. Lutz Damerow

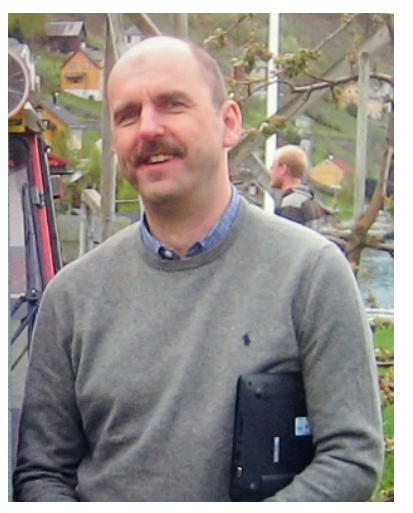


bestimmte er aktiv die Geschicke und Zukunft der Gartenbauwissenschaft mit, insbesondere der Technik im Gartenbau. Über mehrere Jahre war er Sektionssprecher der Technik und Vorstandsmitglied der DGG.

Mit ihm verlieren wir - viel zu früh - nicht nur einen innovativen Vordenker in der Gartenbautechnik, sondern auch einen guten Freund und geschätzten, kooperativen, sympathischen Kollegen.

Unser Mitgefühl gilt seiner Frau und seiner Familie.

Ich werde meinen Freund Lutz Damerow stets in sehr guter Erinnerung behalten.

M. Blanke, Bonn

Funding. Open Access funding enabled and organized by Projekt DEAL.

Open Access. Dieser Artikel wird unter der Creative Commons Namensnennung 4.0 International Lizenz veröffentlicht, welche die Nutzung, Vervielfältigung, Bearbeitung, Verbreitung und Wiedergabe in jeglichem Medium und Format erlaubt, sofern Sie den/die ursprünglichen Autor(en) und die Quelle ordnungsgemäß nennen, einen Link zur Creative Commons Lizenz beifügen und angeben, ob Änderungen vorgenommen wurden.

Die in diesem Artikel enthaltenen Bilder und sonstiges Drittmaterial unterliegen ebenfalls der genannten Creative Commons Lizenz, sofern sich aus der Abbildungslegende nichts anderes ergibt. Sofern das betreffende Material nicht unter der genannten Creative Commons Lizenz steht und die betreffende Handlung nicht nach gesetzlichen Vorschriften erlaubt ist, ist für die oben aufgeführten Weiterverwendungen des Materials die Einwilligung des jeweiligen Rechteinhabers einzuholen.

Weitere Details zur Lizenz entnehmen Sie bitte der Lizenzinformation auf http://creativecommons.org/licenses/by/4.0/deed.de. 